\title{
Thermal and Rheological Characterization of Polyurethanes and Their Blends Having Different Soft Segment Length
}

\author{
Ryuichi URAKAWA ${ }^{*}$, Akira MochizUKi ${ }^{* *}$, and Masaoki TAKAHASHi ${ }^{* * *}$ \\ ${ }^{*}$ Critical Care Merchandise Development Team, TERUMO corporation, \\ 1727-1, Tsuijiarai, Shouwa-machi, Nakakoma-gun, Yamanashi, 409-3853, Japan \\ **Kofu Higashi Factory, TERUMO corporation \\ 1727-1, Tsuijiarai, Shouwa-machi, Nakakoma-gun, Yamanashi, 409-3853, Japan \\ ${ }^{* * *}$ Department of Polymer Science and Engineering, Kyoto Institute of Technology, \\ Matsugasaki, Sakyou-ku, Kyoto, 606-8585, Japan \\ (Received : October 18, 2001)
}

\begin{abstract}
Segmented-polyurethanes (SPUs) having different soft segment length $\left(M_{\mathrm{n}}=530-3,000\right)$ and their blends were characterized by NMR, DSC and dynamic viscoelastic analyses. The SPUs were prepared from the reaction of 4,4'diphenylmethane diisocyanate, 1,4-butanediol and polycaprolactone diol. It has been found from the DSC analysis that the long soft segment SPUs with the long hard segment have two melting points due to the hard segment while the short soft segment SPU with the short hard segment has one melting point. The dynamic viscoelastic analysis reveals that the short soft segment SPU forms a glass-like structure more easily than the long soft segment SPUs. The blends of SPUs with various composition ratios were made from the two SPUs which were different in soft segment length $\left(M_{\mathrm{n}}=530\right.$ and $2,000)$. DSC and viscoelastic analyses of the blends show that the short hard segment strongly affects the formation of the crystalline structure. The short segment in the blend seems to prevent the long segment from forming the longsegment-based crystalline structure, and both the segments apparently form the short-segment-based crystalline structure.
\end{abstract}

Key Words: Segmented-polyurethane / Soft segment / Polyurethane blend / Viscoelastic property / Microphase separation

\section{INTRODUCTION}

Thermoplastic segmented-polyurethane (SPU) is one of the important polymeric materials and used in many medical devices such as catheters. It is well-known that SPU shows excellent biocompatibility ${ }^{1,2)}$ or blood compatibility ${ }^{3), 4)}$, and it has been studied as a material for artificial organs.. ${ }^{5,6)}$ SPU is composed of hard and soft segments. The hard segment generally consists of a diisocyanate such as 4,4'diphenylmethane diisocyanate (MDI) and a low molecular weight diol such as 1,4-butanediol (BD). The soft segment is usually selected from polyether diols, polyester diols and polycarbonate diols with the molecular weight of 500-5,000. Generally, as both the segments are thermodynamically immiscible, they form the microphase-separated structure composed of the hard domain and the soft domain. The hard domain provides both physical crosslink sites and fillerlike

* To whom correspondence should be addressed.

tel : 055-275-7359 fax : 055-275-7364

e-mail : ryuuichi_urakawa@terumo.co.jp reinforcement to the matrix, and the soft domain gives high flexibility at room and low temperatures. Thus SPU has unique mechanical properties such as shape-memory behavior. ${ }^{7), 8}$ To control the property of SPU, designing the domain structure is important. Furukawa et al. synthesized SPUs with regulated hard segment lengths and characterized them. They concluded that the mechanical properties of the SPUs were affected by varying the hard segment length. ${ }^{9}$ Van Bogard et al. investigated the morphological structure and the mechanical properties of SPU consisting of polycaprolactone as a soft segment. They found that the morphological structure was changed by the hard segment length and thermal history. ${ }^{10)}$ A study performed by Qin et al. by using model urethane compounds proved that the chain length affected hard crystalline structure. ${ }^{11)}$

We have investigated SPUs for a catheter, which should have sufficient stiffness at the time of insertion into the blood vessel and become soft after being left in the blood vessel. It is important to control the crystalline structure and rheological 
properties of the SPUs. As mentioned above, both segment lengths affect the morphological structure and the mechanical properties of the SPUs. In this study, blends of the SPUs having different soft segment length were prepared in order to control their properties. The effects of soft and hard segment lengths were investigated on crystallization of hard segments, the crystalline structure and the glass transition temperature of both the SPU and their blends.

\section{EXPERIMENTAL}

\subsection{Materials}

The SPUs were prepared from the reaction of 4,4'diphenylmethane diisocyanate (MDI), 1,4-butanediol (BD) and polycaprolactone diol (PCL) by a general one shot method at molar ratio of $[\mathrm{NCO}] /[\mathrm{OH}]=1$. The molecular weight and the content of PCL in the SPUs are listed in Table I together with their abbreviations.

\subsection{Calculation of the average hard segment length}

The chemical structure of the SPUs is described as [[(MDI$\left.\mathrm{BD})_{\mathrm{n}}-\mathrm{MDI}\right]-\mathrm{PCL}$, where $n$ is the average number of the repeating unit in the hard segment. Since the SPUs were prepared at a molar ratio of $[\mathrm{NCO}] /[\mathrm{OH}]=1$, the number of end groups in each segment is equivalent. Therefore, Eq.(1) presented below is given, where $X$ is the weight fraction of the soft segment calculated from NMR result, and $M$ is the molecular weight of each component.

$$
\frac{X}{M_{\mathrm{PCL}}}=\frac{1-X}{n\left(M_{\mathrm{MDl}}+M_{\mathrm{BD}}\right)+M_{\mathrm{MDI}}}
$$

On the basis of Eq. (1) we define the average hard segment length as follows;

Average hard segment length $=n+1$

$$
=\frac{1}{\left(M_{\mathrm{MDI}}+M_{\mathrm{BD}}\right)}\left[\frac{(1-X)}{X} M_{\mathrm{PCL}}-M_{\mathrm{MDI}}\right]+1
$$

The calculated values of $(n+1)$ are summarized in Table I.

Table I Description of the segmented-polyurethanes (SPUs).

\begin{tabular}{ccccccc}
\hline Designation & \multicolumn{2}{c}{ Soft segment } & & \multicolumn{3}{c}{ Hard segment } \\
\cline { 2 - 3 } \cline { 5 - 7 } & $M \mathrm{n}$ & $\begin{array}{c}\text { Content } \\
(\mathrm{wt} \%)^{\mathrm{a}}\end{array}$ & & $\begin{array}{c}\text { Average hard } \\
\text { segment length }\end{array}$ & $\begin{array}{c}\text { Tm } 1 \\
\left({ }^{\circ} \mathrm{C}\right)\end{array}$ & $\begin{array}{c}\text { Tm2 } \\
\left({ }^{\circ} \mathrm{C}\right)\end{array}$ \\
\hline SPU1 & 530 & 37 & & 2.9 & 169.2 & $-\mathrm{b}$ \\
SPU2 & 1000 & 37 & & 5.3 & 178.6 & 197.4 \\
SPU3 & 2000 & 37 & & 10.3 & 184.0 & 204.7 \\
SPU4 & 3000 & 37 & & 15.3 & 187.9 & 210.0 \\
SPUB & 2000 & 42 & & 12.8 & 181.6 & 202.7 \\
\hline $\begin{array}{l}\text { a: determined by } \\
\text { b: not observed. }\end{array}$ & & & & & & \\
\hline
\end{tabular}

\subsection{Blending and tubing procedures}

The tubes of the SPUs and blends were prepared by using a single-screw extruder at $200-220^{\circ} \mathrm{C}$ in order to evaluate thermal and viscoelastic properties as the catheter shape. Blending was carried out by feeding the different SPUs to the extruder when the tubes were prepared. The pellets of the SPUs were dried in vacuo at $80^{\circ} \mathrm{C}$ for more than $24 \mathrm{~h}$ before extruding. The outer and inner diameters of the tubes were ca. 0.88 and ca. $0.66 \mathrm{~mm}$, respectively. The tubular samples were annealed at $80^{\circ} \mathrm{C}$ for $24 \mathrm{~h}$ to remove internal strain caused by the extrusion before DSC and viscoelastic measurements.

\subsection{NMR analysis}

To examine the molar ratio of the soft segment in the SPU, ${ }^{1} \mathrm{H}-\mathrm{NMR}$ spectroscopy was performed on a NMR spectrometer (Varian Unity Plus 400 ) at $40^{\circ} \mathrm{C}$. Dimethylsulfoxide- $d 6$ was used as a solvent.

\subsection{DSC measurement}

Differential scanning calorimetry (DSC) was carried out with the Shimadzu DSC-50 and operated from -50 to $250^{\circ} \mathrm{C}$ at the rate of $10^{\circ} \mathrm{C} / \mathrm{min}$ under nitrogen atmosphere. The sample weight was ca. $6 \mathrm{mg}$.

\subsection{Viscoelastic measurement}

Dynamic viscoelastic data of the SPUs and the SPU blends were obtained for the samples in the shape of tube with the length of $25 \mathrm{~mm}$ using a dynamic viscoelastometer (DVA-225 ITK. Co. Ltd., Japan). The samples were initially held at $-150^{\circ} \mathrm{C}$ and then heated to $180^{\circ} \mathrm{C}$ at the rate of $5^{\circ} \mathrm{C} /$ min. The data at $10 \mathrm{~Hz}$ were taken at the interval of approximately $1{ }^{\circ} \mathrm{C}$.

\section{RESULTS AND DISCUSSION}

\subsection{Characterization of SPUs}

The effect of soft segment length on the thermal characteristics of the SPUs (SPU1-4) was investigated by using DSC. In this investigation, the molecular weight of PCL corresponding to the soft segment length is changed from 530 to 3,000 , and the content is $37 \mathrm{wt} \%$. With increasing soft segment length, the average hard segment length increases from 2.9 to 15.3 as described in Table I. DSC thermograms of the SPUs (SPU1-4) are shown in Fig.1. The endothermic peaks assigned to the fusion of the crystallite in the hard domain are observed in the range of $160-210^{\circ} \mathrm{C}$, while the melting peak due to the PCL soft domain can not be observed. It is well-known that PCL homo polymer is a crystalline 
polymer and has a melting point at $50-60^{\circ} \mathrm{C}$. These results indicate that the PCL soft segment retains an amorphous state in these SPUs. It is clear from the figure that the soft segment length makes a significant effect on the melting point of the hard domain. That is, the increase of hard segment length due to the increase of the soft segment length brings about the

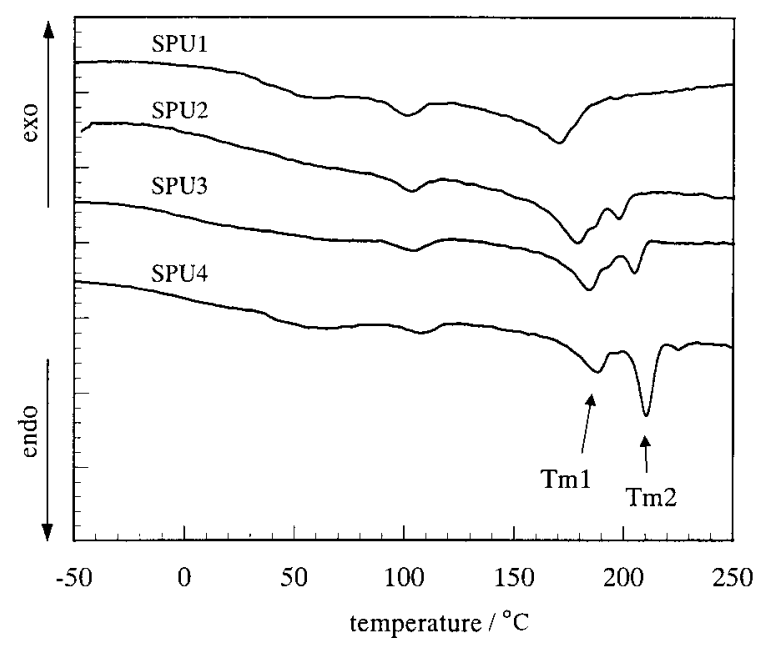

Fig.1 DSC thermograms of SPUs with soft segment content of $37 \mathrm{wt} \%$.
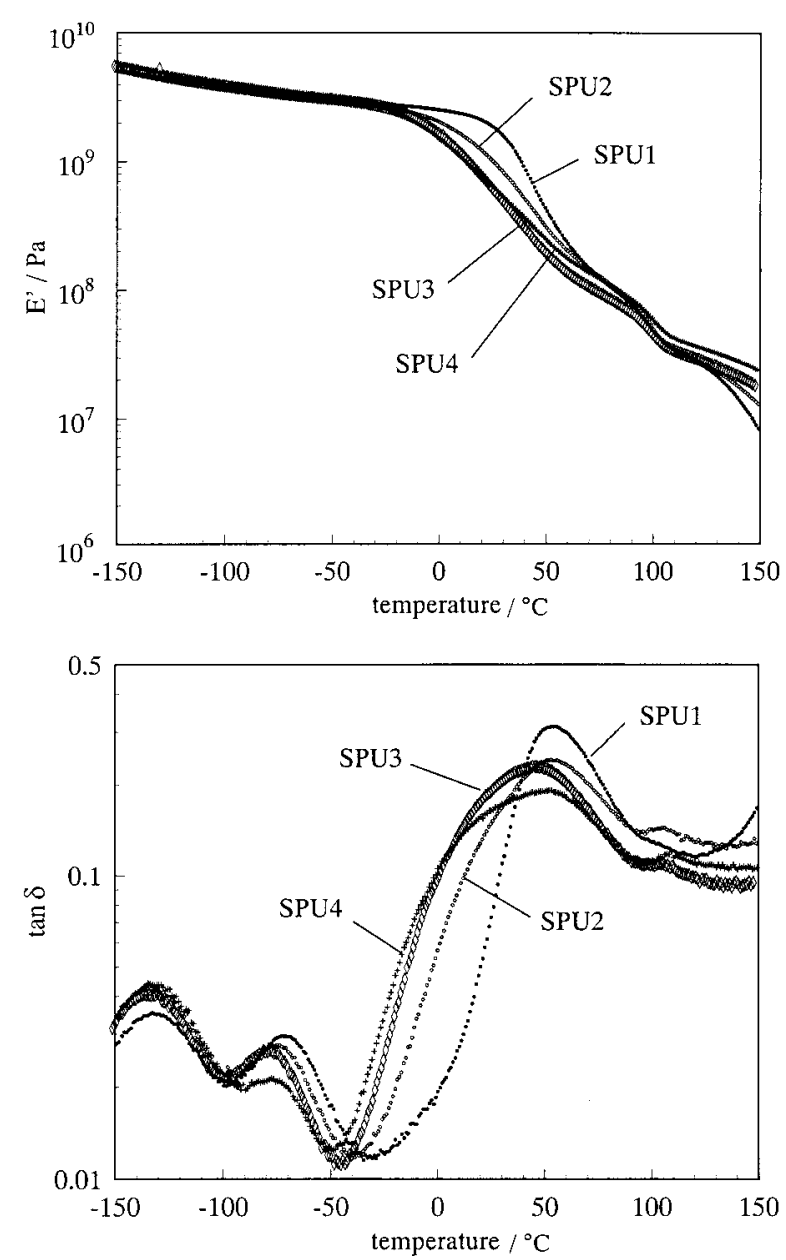

Fig.2 Temperature dispersion curves of $E^{\prime}$ and $\tan \delta$ of SPUs with soft segment content of $37 \mathrm{wt} \%$. formation of a crystalline structure different from the one formed by the short segment. The sample SPU1 composed of the short soft segment $\left(M_{\mathrm{n}}=530\right)$ has only one melting point at $169^{\circ} \mathrm{C}(T m 1)$ while the SPUs (SPU2, SPU3 and SPU4) composed of PCL with the molecular weight of more than 1,000 have two melting points, $\operatorname{Tm} 1$ and $\operatorname{Tm} 2$. In addition, it is found that in these three SPUs both the peaks shift to higher temperatures with increasing hard and soft segment length and that the peak intensities around $\operatorname{Tm} 1$ and $\operatorname{Tm} 2$ have a tendency to decrease and increase, respectively, with increasing hard segment length. The crystalline structure related to $T m 1$ may involve both the hard and soft segments, which are partially miscible. The structure related to $\operatorname{Tm} 2$ may be composed of the more isolated hard segment. This statement is supported by the above-mentioned reports. ${ }^{10), 12), 13)}$ It is considered that the increase of the segment length causes more strong immiscibility between the hard and soft segments.

The endothermic peak appeared at around $100^{\circ} \mathrm{C}$ will be short-range reorganization within a hard microcrystallite, which is induced by annealing at $80^{\circ} \mathrm{C} .^{12)-15)}$

The viscoelastic behaviors of SPUs are shown in Fig.2. The decrease in the storage modulus $E$ ' of SPU1 by glass transition occurs at the highest temperature among the SPUs investigated. That is, the decrease starts at ca. $15^{\circ} \mathrm{C}$, and the moduli of the other samples decrease at ca. $-20^{\circ} \mathrm{C}$. In addition, the $E$ ' of SPU1 decreases rapidly, while those of the other SPUs decrease gradually with increasing temperature. Each $\tan \delta$ curve of the SPUs in the range of -50 to $80^{\circ} \mathrm{C}$ has a maximum at around $50^{\circ} \mathrm{C}$, and the $\tan \delta$ peak of SPU1 is very sharp compared with those of the others. This sharpness in the peak suggests that both segments in SPU1 form more amorphous structure than those in other SPUs. It is considered that the motion of the soft segment chains of SPU1 is strongly restricted by the hard domain, because the soft segment is partially included in the hard domain due to the miscibility. ${ }^{16)}$ That is, the micro-phase-separated structure of the SPU consisted of short segments will not be formed clearly in this sample.

\subsection{Characterization of the SPU blends}

The component SPUs used to blend are SPU1 and SPUB, and the latter is comprised of PCL with $M_{\mathrm{n}}=2000$ (42 wt\%). The characteristics of these SPUs are listed in Table I. The hard segment length of SPUB is about 4 times longer than that of SPU1.

Blending of SPU1 and SPUB was carried out at the composition ratio of $3: 7,5: 5$ and 7:3 (wt/wt) by using the extruder. The DSC thermograms of the components and the 
blends are shown in Fig.3. The SPUB has also two melting points based on the fusion of the hard segment crystallites, $T m 1$ and $T m 2$. The incorporation of SPU1 into SPUB results in the shift of $T m 1$ and $T m 2$ to lower temperatures. The facts that blends obtained are transparent, that the depression of $\mathrm{Tm}$ of PU domain according to the incorporation of soft segment is observed and that the hard and the soft segments in both SPU1 and SPUB have the same chemical structure each other except for the chain length imply that the hard and the soft domains of these SPUs are partially miscible. ${ }^{17), 18)}$ In addition, the peak intensity of $T m 2$ decreases markedly with increasing SPU1 content and the peak finally disappears at the blend ratio of 7:3. On the contrary, the peak intensity of $T m 1$ increases considerably with the increase of the SPU1 content. It is obvious from the figure that the crystalline structure related to $T m 1$ is predominant in the blends regardless of the blend composition. These results indicate that the SPU1 composed of the short hard segment significantly prevents the long hard segment from forming the crystalline structure associated with $T m 2$. The reason for these phenomena may be explained as

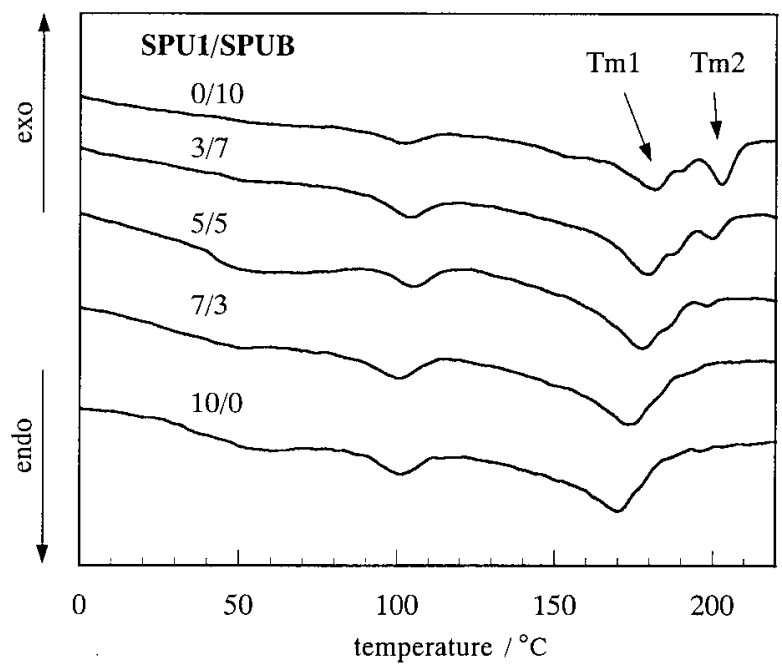

Fig.3 DSC thermograms of SPU blends and the components (SPU1 and SPUB).

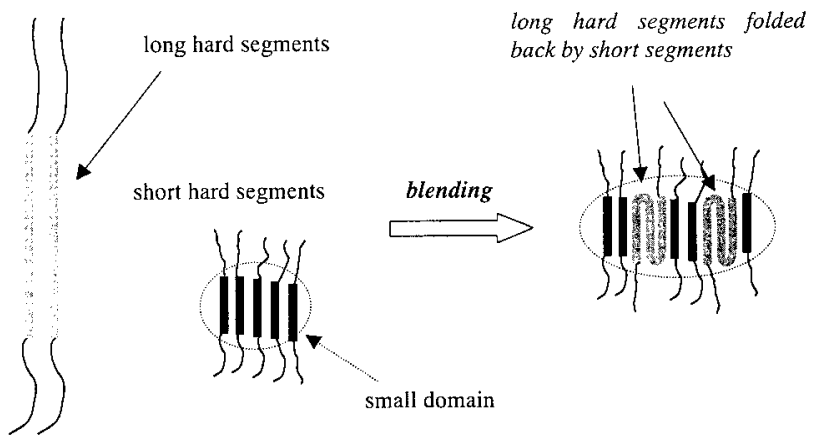

Fig.4 Schematic illustration of crystalline structure in hard domain of SPU blends. follows. The long hard segments will be included into the hard domain composed of the short hard segments by folding back the chains as demonstrated in Fig.4. Thus, the apparent crystalline structure observed in the blends is mainly the one related to $\operatorname{Tm} 1$. A similar folding will also occur in the soft domain.

The dynamic viscoelastic behaviors of the blends are shown in Fig.5. It is found that the decrease in $E^{\prime}$ of the blends shifts to higher temperature with increasing SPU1 content. Especially, even $30 \mathrm{wt} \%$ of the SPU1 content brings about the marked increase of the decreasing point. The blends have similar tan $\delta$ curves below $-50^{\circ} \mathrm{C}$ but they have the different curves in the range of -50 to $100^{\circ} \mathrm{C}$. In the range of -50 to $25^{\circ} \mathrm{C}$, $\tan \delta$ value increases with increasing SPUB content, which is due to the obvious formation of soft domain. The maxima of $\tan \delta$ curves of SPU1 and the blends appear at almost the same temperature around $55^{\circ} \mathrm{C}$. These facts suggest that the long soft segment as well as the long hard segment in the blend folds back, so that the crystalline structure similar to that in SPU1 forms.
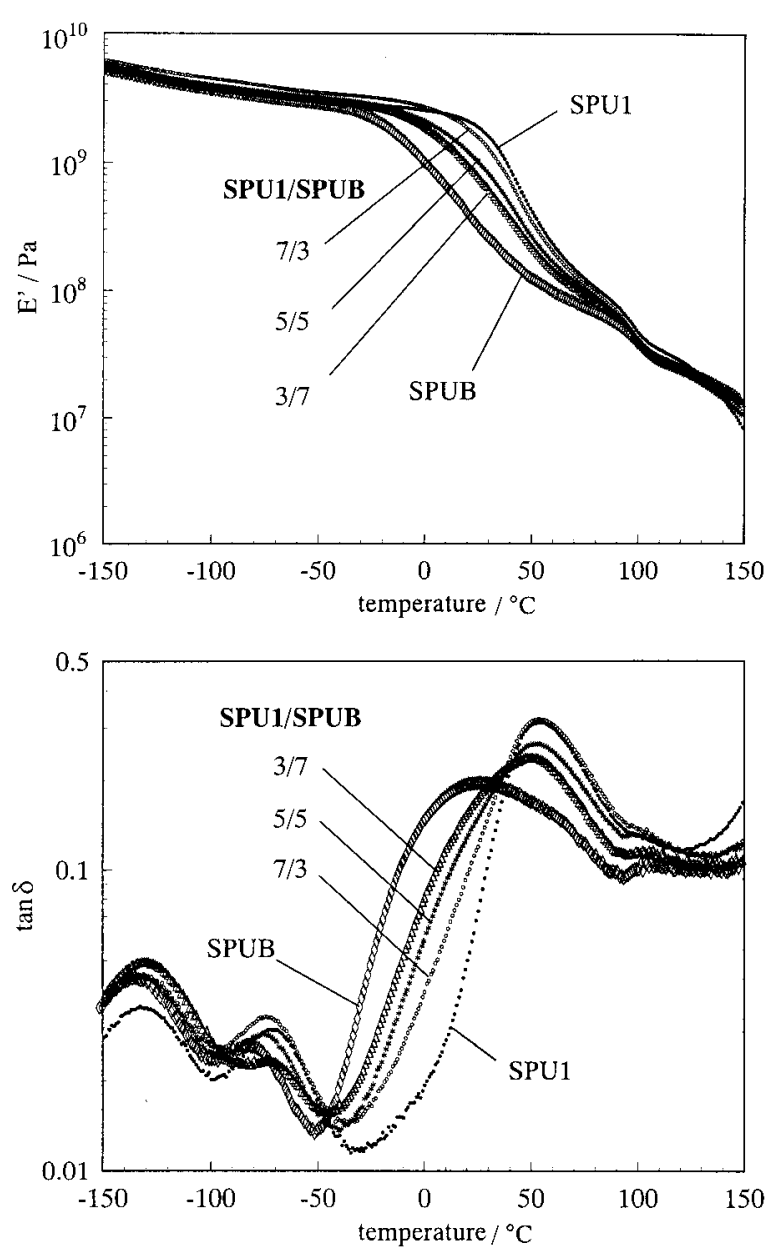

Fig.5 Temperature dispersion curves of $E^{\prime}$ and $\tan \delta$ of SPU blends and the components (SPU1 and SPUB). 


\section{CONCLUSIONS}

The segmented-polyurethanes (SPUs) and the blends composed of SPUs having different soft segment length were characterized by NMR, DSC and dynamic viscoelasticity. The following conclusions are drawn from the studies.

(1) The hard segment length increases with increasing soft segment length.

(2) The SPU with the soft segment (PCL) having $M_{\mathrm{n}}$ more than 1,000 has two melting points ( $T m 1$ and $T m 2$ ) based on the crystallites in the hard domain, while the SPU with the soft segment $M_{\mathrm{n}}=530$ has one melting point ( $\left.\operatorname{Tm} 1\right)$.

(3) In the blends, the number and the intensity of Tm peaks in the DSC thermograms are strongly affected by the composition ratio of the two SPUs.

(4) The SPU composed of the short hard segment in the blend significantly prevents the long hard segment of the other SPU from forming the crystalline structure associated with the long segment.

(5) When the soft segment is short, the motion of the soft segment is strongly restricted by the hard domain because of the partial miscibility of both the segments.

(6) The thermal and the viscoelastic behaviors in the SPU blends are controlled by a factor that the long hard segment and thus also the soft segment are folded back into the short hard segments resulting in the formation of similar domain structure to the original domain formed by the short segment.

(7) The physical properties of SPU blends can be controlled by changing the composition ratio of the SPUs having different segment lengths.

\section{REFERENCES}

1) Marthur A B, Collier T O, Kao W J, Wiggins M, Schubert M A, Hiltner A, Anderson J M, J Biomed Mater Res, 36, 246 (1997).

2) Bakker D, van Blitterswijk C A, Hesseling S C, Koerten H K, Kuijpers W, Grote J J, J Biomed Mater Res, 24, 489 (1990).

3) Kohjiya S, Ikeda Y, Yamashita S, Hayashi K, Yamamoto N, Yamashita I, Nippon Gomu Kyoukaishi, 59, 685 (1986).

4) Takahara A, Tashita J, Kajiyama T, Takayanagi M, MacKnight W J, Polymer, 26, 987 (1985).

5) Ijima H, Nakazawa K, Koyama S, Kaneko M, Matsushita T, Gion T, Shirabe K, Shimada M, Takenaka K, Sugimachi K, Funatsu K, Int J Artif Organs, 23, 446 (2000).

6) Abe $Y$, Chinzei $T$, Isoyama $T$, Ono $T$, Mochizuki S, Saito I, Guba P, Karita T, Sun Y P, Kouno A, Suzuki T, Baba K, Mabuchi K, Imachi K, Int J Artif Organs, 23, 221 (1999).

7) Lin J R, Chen L W, J App Polym Sci, 69, 1563 (1998).

8) Lin J R, Chen L W, J App Polym Sci, 69, 1575 (1998).

9) Furukawa M, Yatake M, Yokoyama T, Nippon Gomu Kyoukaishi, 60, 46 (1987).

10) Van Bogart J W C, Gibson P E, Cooper S L, J Polym Sci: Polym Phys Ed, 21, 65 (1983).

11) Qin Z Y, Macosko C W, Wellinghoff S T, Macromolecules, 18, 553 (1985).

12) Leung L M, Koberstein J T, Macromolecules, 19, 706 (1986).

13) Koberstein J T, Russell T P, Macromolecules, 19, 714 (1986).

14) Stack G M, Beumel L L, J Appl Polym Sci, 44, 305 (1992).

15) Yoon P J, Han C D, Macromolecules, 33, 2171 (2000).

16) Sanchez-Adsuar M S, Pastor-Blas M M, Martin-Martinez J M, Villenave J J, Int J Adhesion and Adhesives, 17, 155 (1997).

17) Kobayashi M, Nishimura H, Oguro K, Kunii N, Int Prog Urethanes, 6, 130 (1993).

18) Kobayashi M, Oguro K, Kunii N, Nishimura H, Reports Res Lab Asahi Garasu Co., Ltd., 39, 99 (1989). 